\title{
Avoided crossings and dynamical tunneling close to excited-state quantum phase transitions
}

\author{
D.J. Nader, ${ }^{1, *}$ C.A. González-Rodríguez, ${ }^{2, \dagger}$ and S. Lerma-Hernández ${ }^{1, \ddagger}$ \\ ${ }^{1}$ Facultad de Física, Universidad Veracruzana, Circuito Aguirre Beltrán s/n, Xalapa, Veracruz 91000, Mexico \\ ${ }^{2}$ Facultad de Ingeniería, Universidad Veracruzana, Coatzacoalcos, Veracruz, México.
}

\begin{abstract}
Using the Wherl entropy, we study the delocalization in phase-space of energy eigenstates in the vicinity of avoided crossing in the Lipkin-Meshkov-Glick model. These avoided crossing, appearing at intermediate energies in a certain parameter region of the model, originate classically from pairs of trajectories lying in different phase space regions, which contrary to the low energy regime, are not connected by the discrete parity symmetry of the model. As coupling parameters are varied, a sudden increase of the Wherl entropy is observed for eigenstates close to the critical energy of the excited-state quantum phase transition (ESQPT). This allows to detect when an avoided crossing is accompanied by a superposition of the pair of classical trajectories in the Husimi functions of eigenstates. This superposition yields an enhancement of dynamical tunneling, which is observed by considering initial Bloch states that evolve partially into the partner region of the paired classical trajectories, thus breaking the quantum-classical correspondence in the evolution of observables.
\end{abstract}

\section{INTRODUCTION}

Semi-classical approximations in phase-space are very useful to gain intuitive insights about the behaviour of quantum systems [1-3]. The correspondence between classical trajectories and phase-space representation of quantum states, together with the knowledge of large (or even whole) energy portions of the classical phasespace dynamics, usually accessible in systems with few degrees of freedom, allow to understand many aspects of the quantum model. For instance, critical phenomena as excited-state quantum phase transitions (ESQPTs) [4] are associated to unstable fixed points appearing in the classical dynamics[5-7], also the exponential growth of outof-time-ordered correlators can be understood from the classical exponential sensitivity to initial conditions [8].

Likewise, crossings and avoided-crossings of energy levels can be studied from a semi-classical perspective. In one-degree of freedom Hamiltonians, as the LipkinMeshkov-Glick (LMG) model studied here, crossings and avoided crossings may appear when there exist different classical trajectories for the same energy. According to the von Neuman-Wigner theorem [9], crossings between states belonging to the same symmetry sector are rather rare and what semi-classically would be expected to be a crossing, becomes an avoided crossing. The relation of this phenomenon with tunneling was established since the seminal papers by Landau and Zener [10,11] and continues to be a topic of current interest [12-14].

In this contribution we use semi-classical phase space methods to shed some light in the avoided crossings that appear at intermediate energies of the LMG model. Among many-body quantum systems, the LMG model has been widely used to test many-body phenomena since

\footnotetext{
* djulian@uv.mx

† carlosgonzalez01@uv.mx

‡ slerma@uv.mx
}

it is one of the simplest models that can be reduced to invariant subspaces with only one degree of freedom. The LMG model was originally proposed to mimic the behavior of closed shell nuclei [15]. However it has been shown that it is useful in other branches of physics like quantum spin systems [16], ion traps [17], Bose-Einstein condensates in double wells [18] and cavities [19], and has also been employed to study quantum phase transitions (QPT) [20-22] and decoherence [23].

The LMG model has a discrete parity symmetry, which is spontaneously broken for large enough couplings at low energy (in some cases also at high energy). This spontaneous breaking yields a quasi degeneracy between pairs of states with different parity[24]. Classically, this is manifested as pairs of trajectories moving in different phase space regions that are connected by the parity transformation. At the critical ESQPT energy, the parity symmetry is restored what is expressed classically as trajectories which are mapped into themselves by the parity transformation. For a particular sector of the parameter space, classically disconnected and no parity-related phase space regions of degenerate trajectories appear at intermediate energy, this manifests in the quantum model as crossings between states of different parity and avoided crossing between states of the same parity for specific values of the coupling parameters.

We use the Wherl entropy (the Shannon entropy of the Husimi function) to measure the phase space localization of energy eigenstates and determine its relation with the avoided crossings and the superposition of classical trajectories appearing at energies close to the ESQPT. Other Shannon entropies in avoided crossing have been discussed in atomic systems [25, 26] and also in billiards [13]. Whereas, the Wehrl entropy itself has been used as a reliable indicator of quantum phase transitions [27] and ESQPTs [6] in several models including the LMG model and for analyzing the transition order to chaos in the kicked Harper map[14]. A first approximation to the study of the Wehrl entropy at the vicinity of avoided 
crossing in the LMG model was reported in Ref. [28], where two kind of singular behaviours were observed: a spike-like maximum and a sudden interchange of localization values between the states involved in the avoided crossings. In the same reference, the relation of this behaviour with exceptional points was also discussed.

In this paper, we also consider the evolution of initial Bloch coherent state located on one of the degenerate classical orbits at intermediate energy. With this, we establish the relation between the Wehrl entropy sudden increase, the enhancement of dynamical tunneling and the consequent breaking of the quantum-classical correspondence in the evolution of several observables, as the survival probability and the expectation value of the population operator.

The article is organized as follows, in section II we review briefly the LMG model, its classical limit and the classification of its parameter space according to the behaviour of the energy density of states and the different trajectories appearing in the classical limit. In section III, the Wehrl entropy is introduced as a measure of delocalization in phase space [29] and our results for the eigenstates' Wehrl entropies are discussed focusing on what happens for states involved in avoided crossings. In section IV, the consequences of the sudden increase of the Wehrl entropy for dynamical tunneling and breaking of the classical-quantum correspondence are analyzed. Our conclusions are given in section $\mathrm{V}$.

\section{THE LIPKIN-MESHKOV-GLICK MODEL}

The Lipkin-Meshkov-Glick Hamiltonian can be written in terms of pseudo-spin operators

$$
\hat{H}=\epsilon_{0} \hat{J}_{z}+\frac{V}{2}\left(\hat{J}_{+}^{2}+\hat{J}_{-}^{2}\right)+\frac{W}{2}\left(\hat{J}_{+} \hat{J}_{-}+\hat{J}_{-} \hat{J}_{+}\right)
$$

which satisfy the $\mathrm{SU}(2)$ algebra, $\left[\hat{J}_{z}, \hat{J}_{ \pm}\right]= \pm \hat{J}_{ \pm}$and $\left[\hat{J}_{+}, \hat{J}_{-}\right]=2 \hat{J}_{z}$. The Hamiltonian commutes with the operator $\hat{J}^{2}$, therefore one can easily perform exact diagonalization in the basis of eigenstates $|J m\rangle$ for a given value of $J$. The LMG Hamiltonian has a parity symmetry associated to the operator

$$
\hat{\Pi}=e^{-i \pi\left(\hat{J}_{z}+J \hat{1}\right)}
$$

with eigenvalues \pm 1 , and which is proportional to a rotation by an angle $\pi$ around the $z$-axis.

For convenience we use the parametrization

$$
\gamma_{x}=\left(\frac{2 J-1}{\epsilon_{0}}\right)(W+V), \quad \gamma_{y}=\left(\frac{2 J-1}{\epsilon_{0}}\right)(W-V)
$$

which allows to write the LMG Hamiltonian as

$$
\hat{H}=\epsilon_{0}\left[\hat{J}_{z}+\left(\frac{\gamma_{x}}{2 J-1}\right) \hat{J}_{x}^{2}+\left(\frac{\gamma_{y}}{2 J-1}\right) \hat{J}_{y}^{2}\right],
$$

where $\hat{J}_{ \pm}=\hat{J}_{x} \pm i \hat{J}_{y}$. For simplicity, from now on we set $\epsilon_{0}$ to the unity.

\section{A. Classical hamiltonian}

A classical Hamiltonian can be derived from the previous quantum operator by considering its expectation value respect to Bloch coherent states [30]

$$
\begin{aligned}
|\alpha\rangle & =\frac{1}{\left(1+|\alpha|^{2}\right)^{J}} e^{\alpha \hat{J}_{+}}|J-J\rangle \\
& =\frac{1}{\left(1+|\alpha|^{2}\right)^{J}} \sum_{m=-J}^{J}\left(\begin{array}{c}
2 J \\
J+m
\end{array}\right)^{1 / 2} \alpha^{J+m}|J m\rangle,
\end{aligned}
$$

where $\alpha$ is a complex number that, when defined in terms of the angular spherical coordinates $\alpha=\tan (\theta / 2) e^{-i \phi}$, leads to

$$
H \equiv\langle\alpha|\hat{H}| \alpha\rangle=\epsilon_{0} J\left[z+\frac{\gamma_{x}}{2} x^{2}+\frac{\gamma_{y}}{2} y^{2}\right],
$$

where

$$
z=-\cos \theta, \quad x=\sin \theta \cos \phi, \quad y=\sin \theta \sin \phi,
$$

thus defining the surface of the Bloch sphere $z^{2}+x^{2}+y^{2}=$ 1. The number of degrees of freedom of the model is two. In terms of the set of canonical variables $z$ and $\phi$, the Hamiltonian reads

$$
H=\epsilon_{0} J\left[z+\frac{1-z^{2}}{2}\left(\gamma_{x} \cos ^{2} \phi+\gamma_{y} \sin ^{2} \phi\right)\right] .
$$

Another convenient set of canonical variables well suited to parametrize the surface of the Bloch sphere is given by variables

$$
Q=\sqrt{2(1-\cos \theta)} \cos \phi, \quad P=-\sqrt{2(1-\cos \theta)} \sin \phi,
$$

which map the surface of the Bloch sphere to a disc of radius two, $\sqrt{Q^{2}+P^{2}} \leq 2$, and are related to the coherent parameter through $\alpha=\frac{Q-i P}{\sqrt{4-\left(Q^{2}+P^{2}\right)}}$. With these coordinates, the south pole of the Bloch sphere is mapped to the center of the disc, the north pole to the disc's perimeter and the sphere's equator corresponds to an inner circle of radius one.

\section{B. Characterization of the LMG parameter space}

The classical Hamiltonian (8) allows to identify the phases of the LMG model according to the properties of its ground-state. The LMG model parameter space can be classified [20] by means of the order parameter $\langle\hat{O}\rangle \equiv\left\langle\hat{J}_{z}+J \hat{1}\right\rangle$ and the ground-state energy $E_{g s}$. For $\gamma_{x}>-1$ and $\gamma_{y}>-1$, the order parameter $\langle\hat{O}\rangle=0$ and $E_{g s}=-J$; whereas $\langle O\rangle=J\left(\gamma_{m}+\gamma_{m}^{-1}\right)$ and 

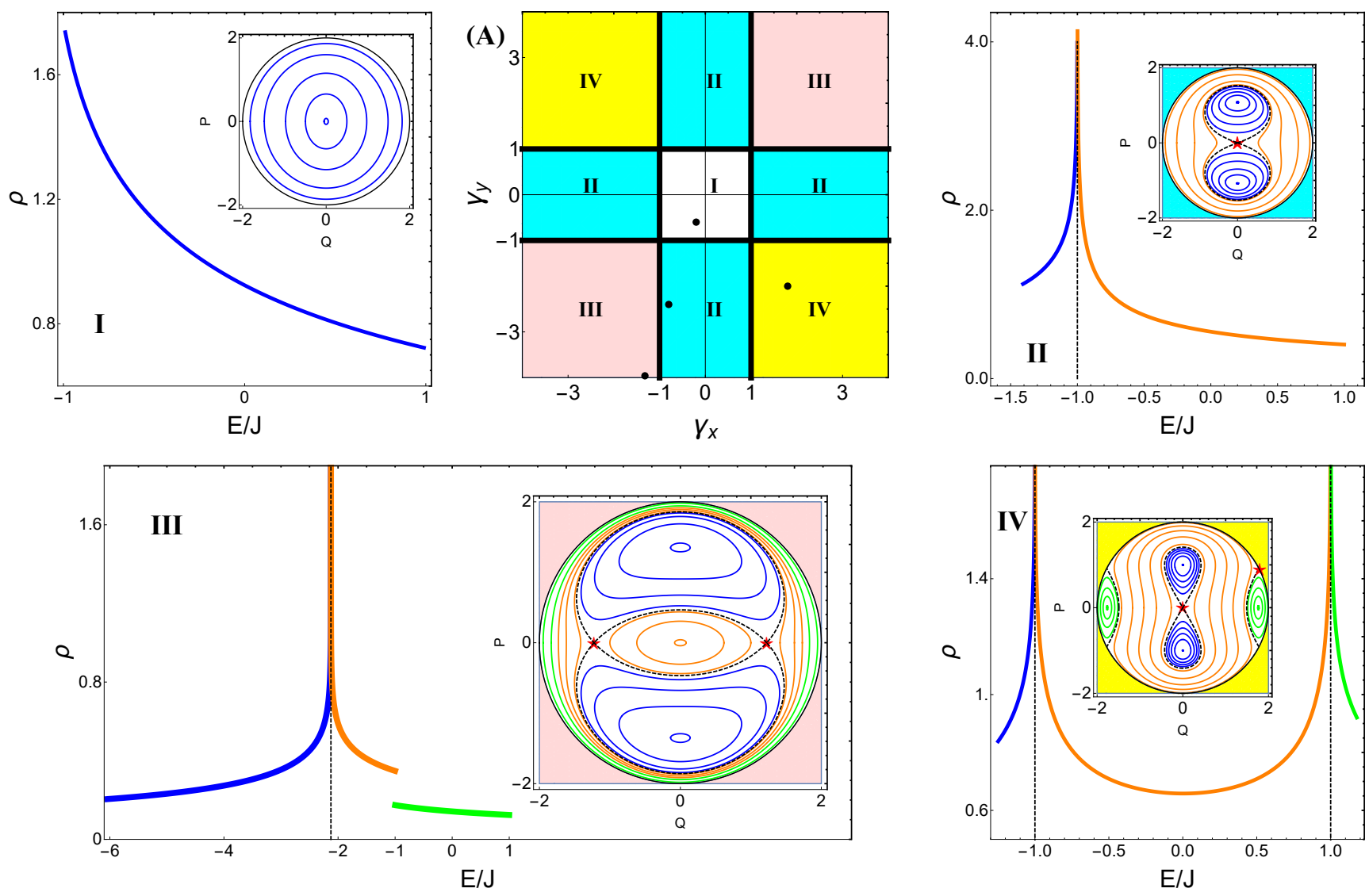

Figure 1: Panel (A) depicts the parameter space of the LMG model classified according to the different semi-classical profiles that can be found for the energy density of states. Typical energy densities of states $(\rho)$ are shown in panels I-IV, the colors of the lines indicate different energy regimes. Inside these panels, typical classical

trajectories are shown with colors specifying their respective energies. In panels II-III and IV the logarithmic divergences in the energy density of states are indicated by vertical lines. The separatrices in phase space associated to these divergences are indicated by dashed lines and hyperbolic fixed points by stars. Dots in panel (A) indicate the cases shown in panels I to IV (to obtain the parameters of panel III the coordinates of the dot have to be scaled by a factor 3 ).

$E_{g s}=\epsilon_{0} J\left(\gamma_{m}+\gamma_{m}^{-1}\right)$ for $\gamma_{x} \leq-1$ or $\gamma_{y} \leq-1$, where $\gamma_{m}=\min \left(\gamma_{x}, \gamma_{y}\right)$. The latter phase is characterized by a spontaneous breaking of the parity symmetry. By looking at the entire spectrum of the model, a richer phase diagram is obtained [22]. Four different sectors in parameter space appear, which are characterized by their distinctive energy density of states (EDoS).

The EDoS can be approximated semiclassically as

$$
\rho_{s c}(E)=\frac{J}{2 \pi} \int d z d \phi \delta(H(z, \phi)-E)
$$

whose explicit evaluation is presented in appendix B. These sectors and representative energy densities of states are depicted in Fig. 1. The sector around the noninteracting case $\left(\left|\gamma_{x}\right|<1\right.$ and $\left|\gamma_{y}\right|<1$, labelled as I in Fig.1) presents a simple EDoS which behaves monotonically as a function of energy. The other three sectors present singularities in the EDoS. In sector II $\left(\left|\gamma_{x}\right|<1\right.$ with $\left|\gamma_{y}\right|<1$ or $\left|\gamma_{y}\right|>1$ with $\left.\left|\gamma_{x}\right|<1\right)$ a logarith- mic divergence is observed at $E / J=-1$ or $E / J=1$ depending on the sign of the coupling with larger absolute value. In sector IV $\left(\left|\gamma_{x}\right|>1,\left|\gamma_{y}\right|>1\right.$ with different signs for $\gamma_{x}$ and $\gamma_{y}$ ) two such divergences occur at $E / J=-1$ and $E / J=1$. Finally, at sector III $\left(\left|\gamma_{x}\right|>1\right.$, $\left|\gamma_{y}\right|>1$ with same signs for $\gamma_{x}$ and $\gamma_{y}$ ), besides a logarithmic divergence occurring at $E=J\left(\gamma_{M}+\gamma_{M}^{-1}\right)$ [with $\left.\gamma_{M}=\operatorname{sgn}\left(\gamma_{x}\right) \min \left(\left|\gamma_{x}\right|,\left|\gamma_{y}\right|\right)\right]$, a discontinuity is observed at $E / J=\operatorname{sgn}\left(\gamma_{x}\right)$. The observed logarithmic singularities in the EDoS define what is called Excited-State Quantum Phase Transitions (ESQPT) for one-degree of freedom systems [31]. The logarithmic divergences are consequence of hyperbolic fixed points in the underlying classical system, which in turn are associated to separatrices of the classical dynamics. The separatrices for typical phase space portraits of sectors II, III and IV are indicated in the panels of Fig. 1 by dashed lines. Observe that in the case of sector III, the separatrix contains two hyperbolic fixed points, differently to the separat- 
(a)

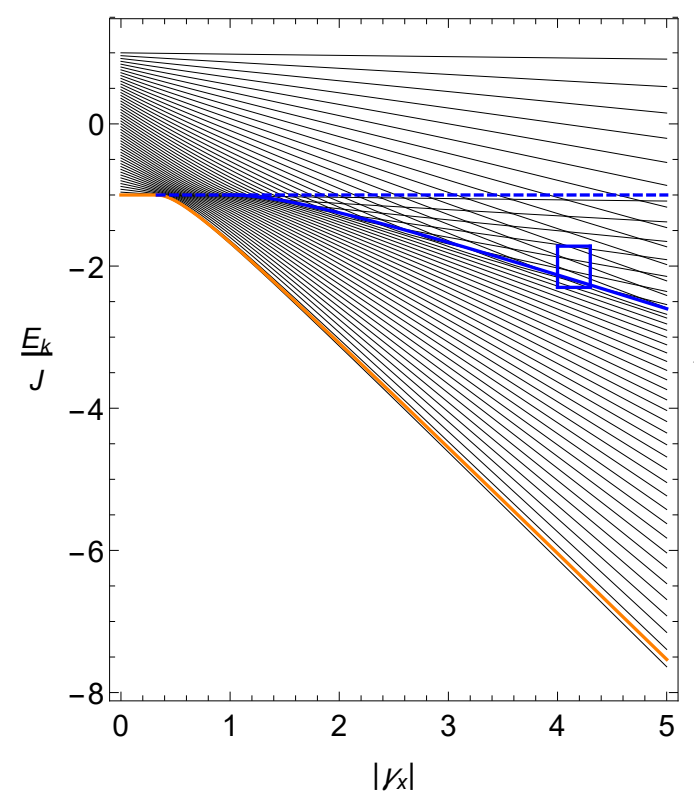

(b)

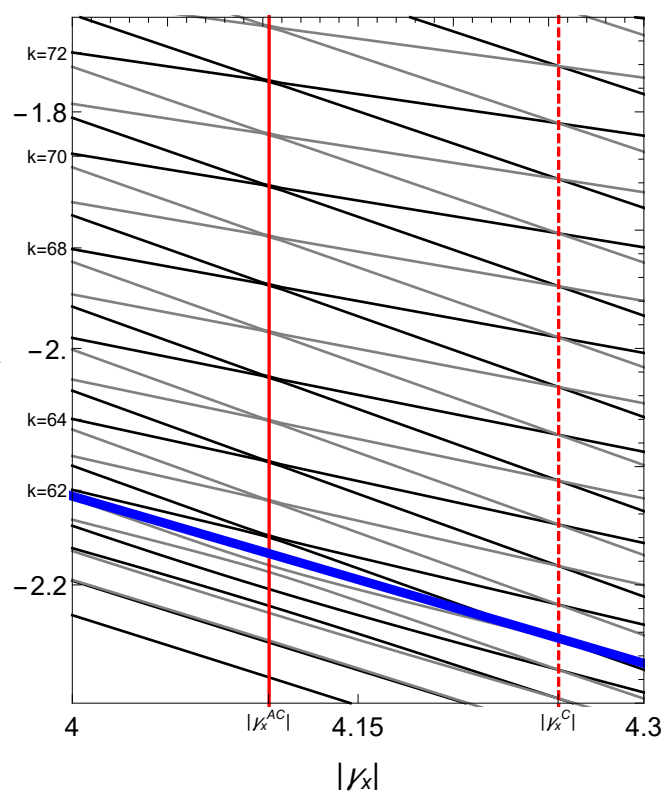

(c)

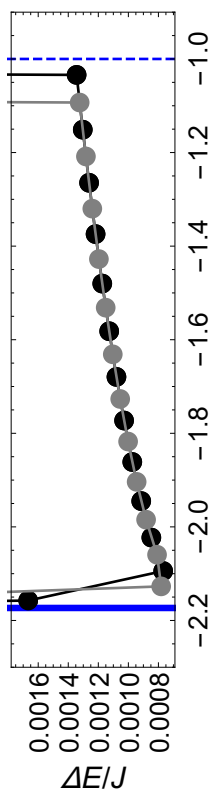

Figure 2: Energy spectrum as a function of coupling parameter $\left|\gamma_{x}\right|$ with $\gamma_{x}<0$ and $\gamma_{y}=3 \gamma_{x}$. Panel (a) shows the whole positive parity spectrum for $J=50$. Color lines correspond to the classical energies for the ground-state (orange), ESQPT energy (blue) and $E / J=-1$ (dashed) where a discontinuity in the EDoS occurs. Panel (b) is a zoom into the rectangle of panel (a), where avoided (vertical solid line) and real (vertical dashed line) crossings take place. For panel (b) a larger $J=100$ was used and the spectrum include positive parity (black lines) and negative parity (gray lines) states. Vertical lines were evaluated with Eq.(11) using $N=172$ (even) for the avoided-crossings condition $\left(\left|\gamma_{x}^{A C}\right|=4.10331\right)$ and $N=173$ (odd) for the real crossings $\left(\left|\gamma_{x}^{C}\right|=4.25529\right)$. Indexes $k$ of some positive-parity states are indicated at the left axis of panel (b). Panel (c) shows the energy difference between levels in avoided crossings of positive (black dots) and negative (gray dots) parity states along the solid vertical of panel (b).

ices of the other sectors which possess only one. On the other hand, the discontinuity in the EDoS observed in sector III has to do with a local maximum (at coordinates $Q=P=0$ ) in the classical energy surface which mark the end of the family of central orange trajectories shown in panel III of Fig.1. Typical trajectories of the classical Hamiltonian are also shown for other energy regimes and regions in parameter space. The energy of each trajectory is indicated by the same colour code used for plotting the EDoS and the variables $Q-P$ are used to represent the Bloch sphere.

In this article we focus on the parameter sector III of Fig. 1 with $\gamma_{x}, \gamma_{y}<0$, i.e., in the region where the EDoS exhibits a logarithmic divergence at $E / J=\left(\gamma_{M}+\gamma_{M}^{-1}\right)$ (with $\gamma_{M}=-\min \left(\left|\gamma_{x}\right|,\left|\gamma_{y}\right|\right)$ and a discontinuity at $E / J=-1$. Classical trajectories with energies between these two critical energies are shown by orange lines in panel III of Fig 1. These trajectories come in degenerate (same energy) pairs formed by an inner trajectory in the center of the phase space and a second trajectory in the outer region. Notice that differently to the pairs of degenerated blue trajectories at low energy, at intermediate energies the trajectories are invariant under the parity transformation of Eq.(2) (a $\pi$ rotation around a perpendicular axis through the origin in the plane $Q-P)$. We will show, next, that these pairs of degenerate classical trajectories are manifested in the quantum model as avoided and reals crossings of energy levels.

\section{Crossings and avoided crossings}

The semi-classical formula for the EDoS (10) describes the trend of the energy spectrum, but it is blind to details as avoided and real crossings that may appear in the spectrum. Fig. 2 shows the exact spectrum as a function of coupling $\left|\gamma_{x}\right|$ for $\gamma_{y}=3 \gamma_{x}$. By varying $\gamma_{x}$ from 0 up to $\gamma_{x}=-5$, sectors I, II and III are traversed, which is reflected by changes in the density of states that can be appreciated in the energy levels. Panel (b) in Fig. 2 shows a zoom into the intermediate energy interval for couplings in the sector III of interest. The lines clearly show simultaneous crossings between states with different parity (dashed vertical line) and avoided crossing between states of the same parity (solid vertical line). The energy gaps of the avoided crossings, hardly visible in panel (b), are depicted in panel (c), which also shows that gaps increase as energy does. 

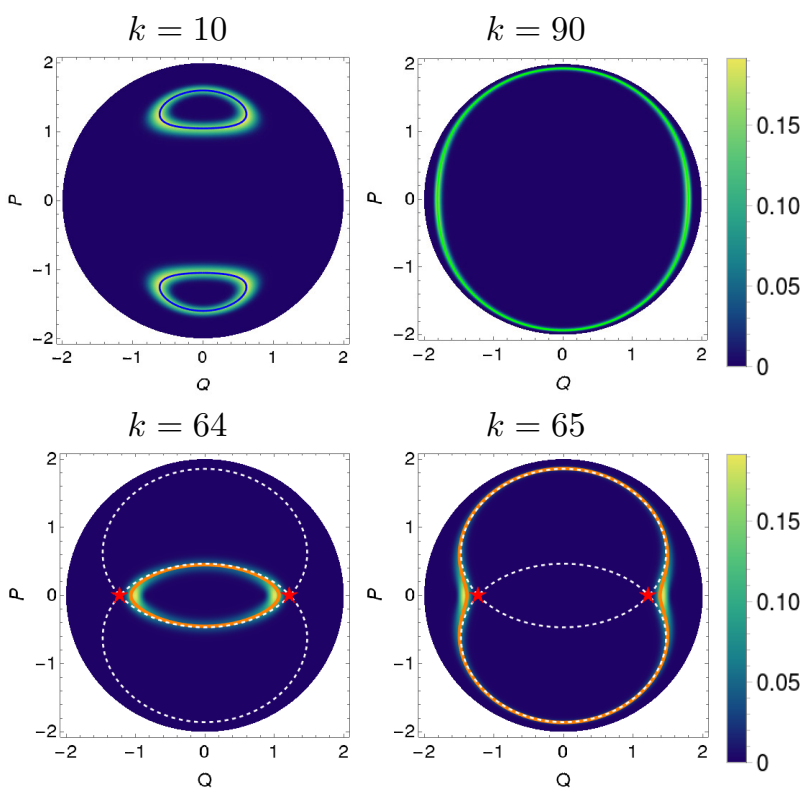

Figure 3: Density plots of Husimi functions $\mathcal{Q}_{k}(\alpha)$ for different eigenstates at $\gamma_{x}=-4\left(\gamma_{y}=3 \gamma_{x}\right)$ with

$J=100$. Solid lines are classical trajectories. For states $k=64$ and $k=65$, dashed lines depict classical separatrices with associated hyperbolic fixed points indicated with stars.

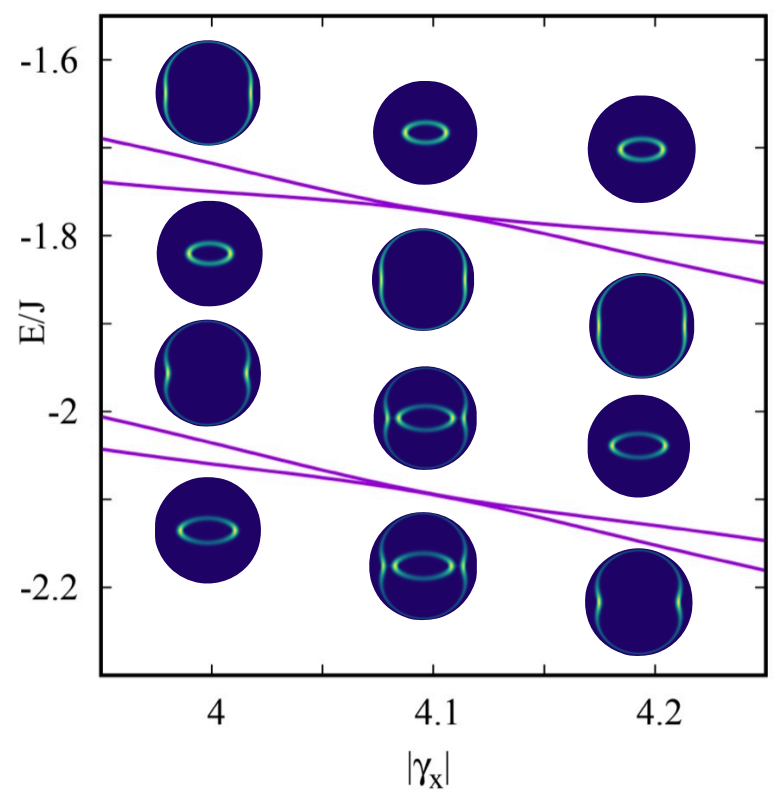

Figure 4: Density plots of Husimi functions $\mathcal{Q}_{k}(\alpha)$ in the vicinity of avoided crossings $\left(\gamma_{x}^{A C}=-4.10331\right.$, with $\gamma_{y}=3 \gamma_{x}$ ) between states $k=64-65$ (bottom), and $k=72-73$ (top), located along the vertical solid line of Fig. 2(b) .
From the Einstein-Brillouin-Keller (EBK) quantization rule, it is possible to determine the values of the coupling constants where crossings or avoided crossing take place at the intermediate energy regime. As mentioned above, at this energy region, there exist, for a given energy, two disconnected classical trajectories $z_{ \pm}(\phi, E)$. From the properties of these degenerate trajectories, we derive (see Appendix B) the condition for crossings (C) and avoided crossings (AC), which reads

$$
\gamma_{x}^{C(A C)} \gamma_{y}^{C(A C)}=\left(\frac{2 J-1}{2 J-N_{\mathrm{o}(\mathrm{e})}}\right)^{2}
$$

with $N_{\mathrm{o}(\mathrm{e})}$ an integer satisfying $0<N_{\mathrm{o}(\mathrm{e})}<2 J$. We have numerically verified that, indeed, for couplings satisfying this condition, crossings between states of different parity take place for $N_{\mathrm{o}}$ odd, whereas for $N_{\mathrm{e}}$ even the crossings predicted by the semi-classical EBK rule become avoided crossing between pairs of states with the same parity. Condition (11) was already reported in Ref.[32] and also in Ref.[33] from analyzing the LMG model as an integrable Richardson-Gaudin model.

In the next section we present a phase-space study of the avoided crossings discussed here. We will use the Husimi function to represent the quantum states. The Husimi function allows not only to study the classicalquantum correspondence but also to measure the delocalization of the states in phase space through the Wehrl entropy [34].

\section{LOCALIZATION MEASURES IN PHASE SPACE}

\section{A. Husimi function and localization measures}

The Husimi representation of a pure state is nothing but its squared projection on the coherent states (5). For eigenstates of the Hamiltonian (1) they are

$$
\mathcal{Q}_{k}(\alpha)=\left|\left\langle\alpha \mid E_{k}\right\rangle\right|^{2}
$$

In Fig. 3, we show the Husimi representation of some eigenstates using the canonical variables $Q-P$ of Eq. (9). We select four positive parity eigenstates in the parameter region III of Fig.1 $\left(\gamma_{x}=-4\right.$ and $\left.\gamma_{y}=3 \gamma_{x}\right)$. The eigenstates with indexes $k=10$ and $k=90$ sit, respectively, in the low energy region of blue trajectories and in the high energy region of green trajectories of panel III in Fig.1, while eigenstates $k=64$ and $k=65$ have intermediate energy in the region of degenerate orange trajectories. We can observe that the Husimi representations are distributed along the respective classical trajectories corresponding to the energy of each state $H=E_{k}$. The intensity of the Husimi function reflects the dynamical properties of the classical trajectory: the Husimi function is more intense in the regions where the classical dynamics is slower. This is clearly seen in states $k=64$ 

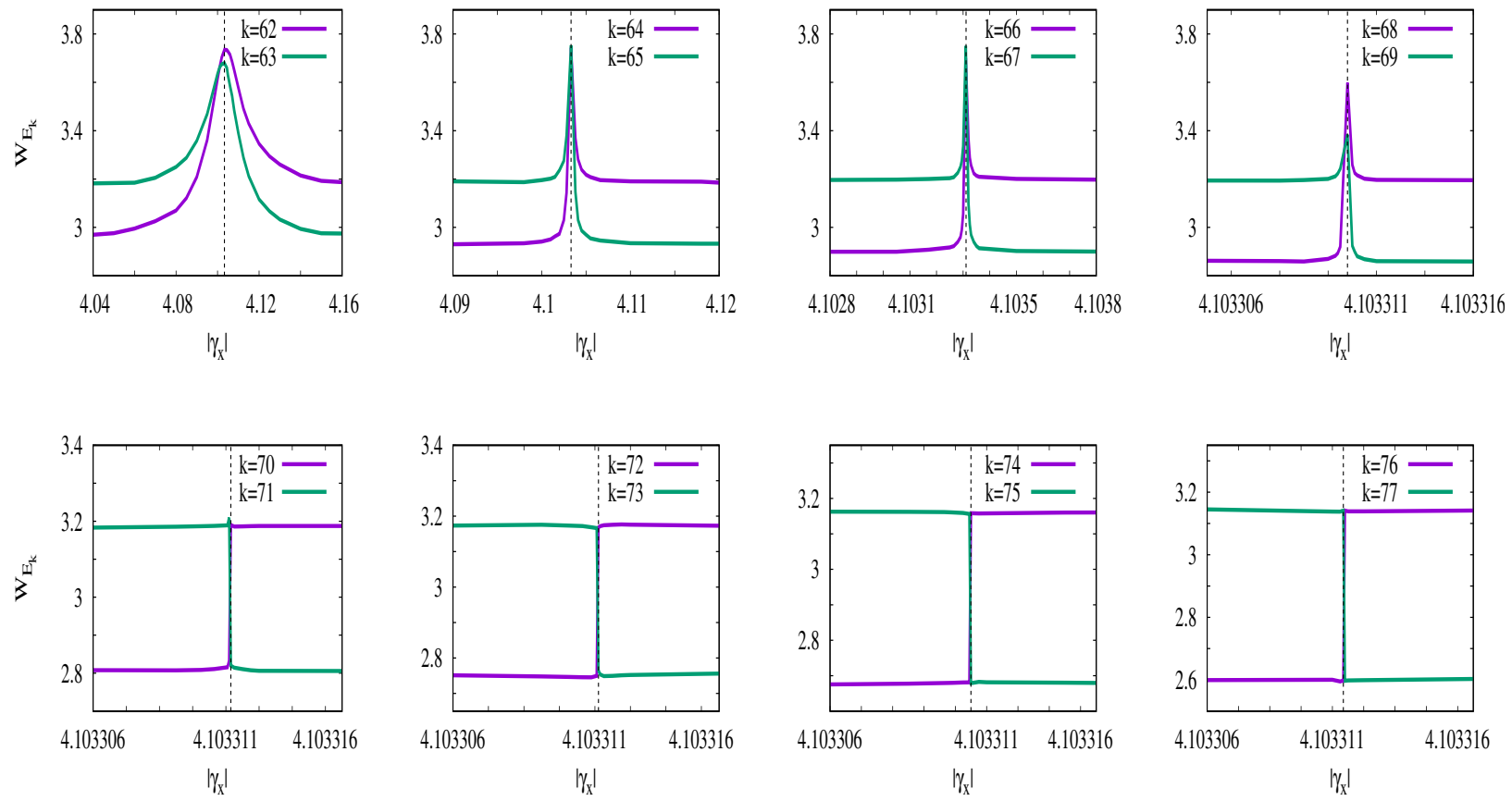

Figure 5: Wherl entropy $W_{E_{k}}$ as a function of the coupling parameter $\left|\gamma_{x}\right|\left(\gamma_{y}=3 \gamma_{x}\right)$ for several states involved in the avoided crossings shown along the solid vertical line of Fig. 2(b). The dashed vertical lines indicate the position of the avoided crossings at $\left|\gamma_{x}\right|=4.1033108$. Different horizontal scales were used in the panels.

and $k=65$ whose dynamics become slow close to the position of the indicated hyperbolic fixed points located, respectively, at $(Q= \pm 1.225, P=0)$.

Fig. 4 focuses on the Husimi functions of states involved in two different avoided crossings of those occurring along the vertical solid line in Fig.2 (b). In both avoided crossing, one can notice that there is an exchange of the Husimi representation between the two levels right after the avoided crossing. However, it is noticeable that in the case of the avoided crossing with energy closer to the ESQPT energy (bottom), one can observe superpositions in the Husimi functions of the two avoided states. These superpositions are located atop of the two classical trajectories with the same energy. Such superpositions occurs only for avoided crossings at energies close to the ESQPT critical energy. For the avoided crossings at higher energies we only observe the exchange in the Husimi representation but not the superposition at the avoided crossing, as illustrated by top avoided crossing in Fig.4.

The superposition of the two classical trajectories in the Husimi functions entails a delocalization in phase space of the eigenstates involved in the avoided crossings. To measure this delocalization, any Rényi entropy in phase space can be employed [35]. Here we use one of the most known, the Wehrl entropy (the Rény entropy of order one) which in our case is given by

$$
\begin{aligned}
W_{E_{k}} & =-\int \mathcal{Q}_{k}(\alpha) \ln \mathcal{Q}_{k}(\alpha) d \Omega \\
& =-\int \mathcal{Q}_{k}(Q, P) \ln \mathcal{Q}_{k}(Q, P) d Q d P
\end{aligned}
$$

where $d \Omega$ is the solid angle of the Bloch coherent states and in the second line we have written the integral in terms of variables $Q-P$ of Eq. (9).

\section{B. Wehrl entropy and avoided crossings in the LMG model}

The Wehrl entropy (13) of several pairs of states involved in avoided crossing are plotted in Fig. 5 as a function of $\gamma_{x}$ (with $\gamma_{y}=3 \gamma_{x}$ ) for intervals around the coupling satisfying the condition for avoided crossings $\gamma_{x}^{A C} \gamma_{y}^{A C}=\left(\frac{2 J-1}{2 J-N}\right)$ with $J=100$ and $N=172$. The same coupling indicated by the solid vertical line in Fig. 2(b). As it was reported in [28] we can notice that there is an exchange in the entropy corresponding to each pair of states involved in the avoided crossings. However, two distinctive kind of exchanges can be observed, depending on the energy of the pair of levels. For avoided crossings with lower energy which are closer to the ESQPT critical energy, the exchange is accompanied by an abrupt rise and fall of the Wehrl entropy. The lo- 

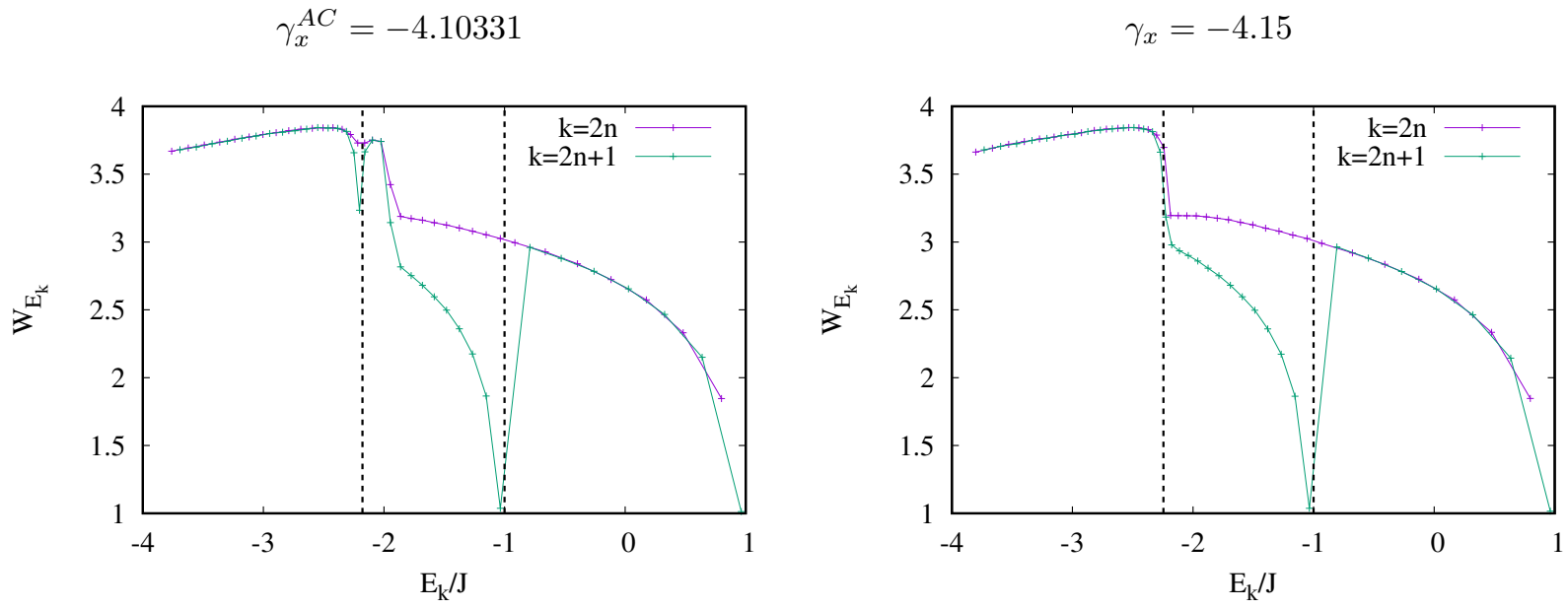

Figure 6: Wehrl entropy as a function of the energy $E_{k}$ for the whole set of parity positive eigenstates. In left panel the parameter $\gamma_{x}$ is chosen at the avoided crossings condition while in the right panel the parameter $\gamma_{x}$ is chosen away of this condition. In both cases $\gamma_{y}=3 \gamma_{x}$ and $J=100$ as in Fig. 2(b). The vertical dashed lines indicate the ESQPT and $E=-1$, respectively. Colors are used to distinguish states with index $k$ even (pink) and odd (green).

cal maxima of the Wehrl entropy diminish and the peaks become narrower as we move away from the ESQPT energy and higher-energy avoided crossings are considered. In Fig. 5, the spike-like behaviour of the Wehrl entropy is clearly seen in the avoided crossing of levels $k=62-63$ up to $k=68-69$, while for the avoided-crossing of levels $k=70-71$ the spike is barely visible. For the rest of avoided crossing the spike has completely disappeared. The sudden increases in the entropy observed for the set of avoided crossings close to the ESQPT energy are direct manifestations of the superposition in the Husimi functions, as the one shown at the bottom of Fig. 4 .

In order to have a broader picture of the behaviour of the Wherl entropy, we show in Fig. 6 the Wherl entropies of all the parity positive eigenstates $\left|E_{k}\right\rangle$ for two different couplings. Left panel corresponds to the same coupling $\gamma_{x}^{A C}$ considered before, satisfying the condition of avoided crossings, and right panel is for a coupling away of this condition. To better visualize the behaviour of the Wherl entropy we use different colors for states with even and odd indexes $k$. The behaviours of the Wherl entropy in the two couplings show some similarities: 1) for energy states below the ESQPT energy (which is indicated by leftmost vertical dashed line), even and odd indexed states follow the same trend with Wehrl entropy increasing as a function of energy; 2) a change in the behaviour of the Wherl entropy is observed for states approaching the ESQPT energy; 3) for states between the two vertical lines and energy far enough of the ESQPT energy, the even and odd labelled states accommodate in two different lines decreasing at different rates, those with odd indexes, whose Husimi functions concentrate around inner classical degenerate trajectories, have lower Wehrl entropy than even indexed states, these latter ones have Husimi functions that concentrate around the outer classical trajectories; 4) since the inner trajectories disappear classically above $E / J=-1$ (energy indicated by the rightmost vertical line), the low line of Wherl entropy values disappear for large energies, and only the upper line remains at energies above $E / J=-1$.

However, striking differences in the behaviour of the Wherl entropy between the two couplings can be observed for states close to the ESQPT energy. For coupling satisfying the condition of avoided crossings, states with energies just above the ESQPT energy have higher Wehrl entropies than the corresponding states of right panel. These higher Wehrl entropy values are the same as those in the peaks of all the plots in top row of Fig. 5 and come from the superposition of the two degenerate classical trajectories that take place in the avoided crossings. These states with higher Wehrl entropies disappear as energy is further increased. We conclude that the avoided crossings observed in the spectrum lead to a superposition of classical degenerate trajectories but only for a small number of states above the ESQPT energy.

\section{PHYSICAL MANIFESTATIONS OF THE WEHRL ENTROPY INCREASE}

\section{A. Enhancement of dynamical tunneling}

In this section we will show that the superposition of degenerate classical trajectories that is observed in the avoided crossings of states just above the ESQPT energy yields an enhancement of dynamical tunneling between regions of the phase space associated to the pair of degenerate classical trajectories.

To this end we consider initial Bloch coherent states centered in a point $Q, P$ on one of the degenerate classical 

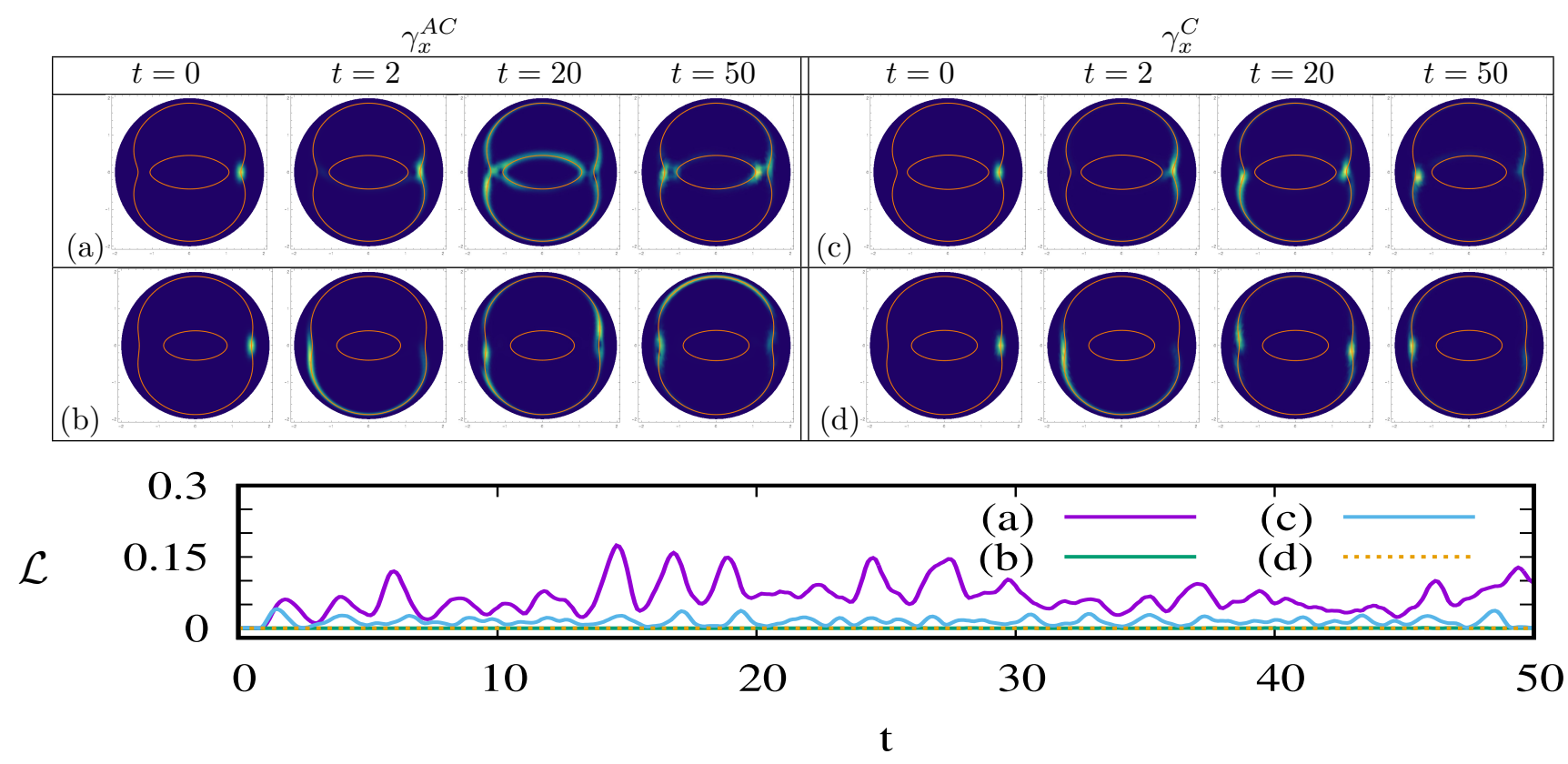

Figure 7: Density plot of the evolution of the Husimi functions $\mathcal{Q}_{\alpha_{0}}(\alpha, t)$ at selected times $t$ for four different initial Bloch coherent states. Solid orange line are classical trajectory at the same energy of the respective initial state

$\left\langle\alpha_{o}|\hat{H}| \alpha_{o}\right\rangle=H$. In panels (a) and (b) the coupling parameters are chosen at the avoided crossing condition $\gamma_{x}^{A C}=-4.10331$ (with $\gamma_{y}^{A C}=3 \gamma_{x}^{A C}$ and $J=100$ ). In panel (a) the initial state has an energy just above the ESQPT and in (b) the initial state has energy in the intermediate regime but far enough from the ESQPT. In panels (c) and (d) the energies of the initial coherent states are chosen similarly to (a) and (b), respectively, but the coupling parameter is far from the condition of avoided crossings $\left(\gamma_{x}^{C}=-4.25529\right.$ with $\left.\gamma_{y}=3 \gamma_{x}\right)$. Bottom row: line integral $\mathcal{L}$ of the Husimi distribution (15) along the partner inner classical trajectory as a function of the time for the same initial states as in panels (a)-(d).

trajectories and study its unitary evolution. Fig. 7 shows the evolution of the Husimi function for such initial states

$$
\mathcal{Q}_{\alpha_{o}}(\alpha, t)=\left|\left\langle\alpha|\hat{U}(t)| \alpha_{o}\right\rangle\right|^{2},
$$

where $\hat{U}(t)$ is the time evolution operator. Panel (a) shows a case when the couplings satisfy the condition of avoided crossings and the initial state has an energy $E_{\alpha_{o}}=\left\langle\alpha_{o}|\hat{H}| \alpha_{o}\right\rangle$ slightly above the ESQPT critical energy. Panel (b) shows a case with the same couplings but for an initial state with larger energy. Panels (c) and (d) show similar initials states with energies just above the ESQPT energy and a larger one, but for couplings not satisfying the condition for avoided crossings. Only in the case of panel (a), the Husimi function, initially located in the outer trajectory, evolves into the inner region of the phase space where the partner degenerate classical trajectory of the initial state is located. In the rest of panels, the Husimi function spreads only along the outer trajectory of the initial state.

In summary, if the initial coherent state is located atop of one of the paired trajectories (inner/outer), its Husimi distribution hoop from the initial trajectory to its pair (outer/inner) if the coupling parameters satisfy the avoided crossing condition (11) and if the energy of the initial state is close to the ESQPT critical energy. In figure 7(a), we show the hooping from the outer to the inner region. In Appendix E, the complementary case is shown, where the hopping occurs from the inner to the outer trajectory.

In order to quantitatively confirm the dynamical tunneling into the partner region, we consider the line integral of the evolved Husimi function along a classical trajectory

$$
\mathcal{L}\left(O_{i}, \hat{U}(t)\left|\alpha_{o}\right\rangle\right)=\oint_{O_{i}} \mathcal{Q}_{\alpha_{o}}(\alpha, t) d l
$$

where $O_{i}$ is the partner classical trajectory of the trajectory where the state is initially located. In the last row of Fig. 7 the line integral (15) is plotted as a function of time for the same initial coherent states as in panels (a) to (d). One can notice that the line integral (15) is significantly larger for state (a), implying that, indeed, dynamical tunneling occurs for coupling parameters at the avoided crossing condition but only for sates having energy close to the ESQPT. 


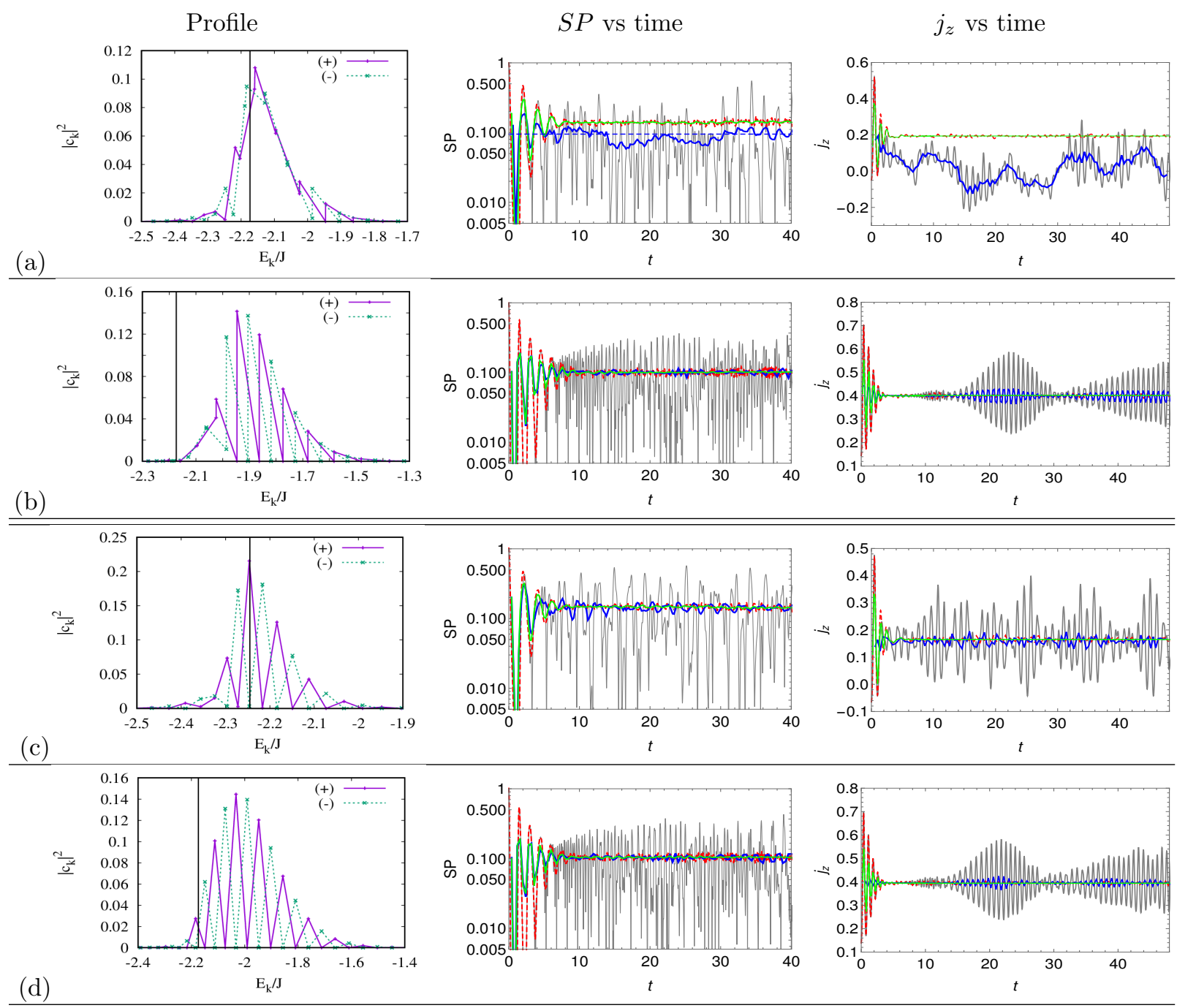

Figure 8: Profiles of energy components (left column), survival probability $(S P)$ in logarithmic scale as a function of time (central column) and temporal evolution of the expectation value $j_{z}$ (right column) for the same initial coherent state as in Fig. 7. In left column, components from positive parity states $(+)$ in pink solid lines and contributions from negative parity states (-) in dashed green lines; the position of the ESQPT is marked with a solid vertical line. In central and right columns, the quantum results correspond to gray light lines and classical results to red dashed lines. Rolling time averages are also included: blue for quantum results and green for classical ones.

\section{B. Evolution of observables and breaking of the classical-quantum correspondence}

Being the tunneling a purely quantum effect, it may manifest as deviations in the temporal evolution of observables respect to the evolution calculated from a classical approximation. In this section, we will show that for initial coherent states, with energy slightly above the ESQPT and for couplings satisfying the condition of avoided crossings, the classical-quantum correspondence for the evolution of observables is broken. In contrast, if the previous conditions are not fulfilled, a remarkable accord between classical and quantum results is obtained.

As observables, we consider the survival probability
(SP) or fidelity,

$$
S P(t)=\left|\left\langle\alpha_{0}|\hat{U}(t)| \alpha_{0}\right\rangle\right|^{2},
$$

and the temporal evolution of the population operator

$$
j_{z}(t) \equiv \frac{\left\langle\hat{J}_{z}\right\rangle(t)}{J}=\frac{1}{J}\left\langle\alpha_{0}\left|\hat{U}^{\dagger}(t) \hat{J}_{z} \hat{U}(t)\right| \alpha_{0}\right\rangle .
$$

As described in Ref. [36] and Appendix D, both quantities can be evaluated classically by using the classical equations through the so called Truncated Wigner Approximation (TWA).

In Fig. 8 we show the results for couplings and same four initial Bloch coherent states as in Fig. 7. The first 
two rows correspond to couplings satisfying the condition of avoided crossings, with the first row showing results for a coherent state with energy slightly above the ESQPT and the second row for a state with larger energy. Last two rows show results for couplings away from the condition of avoided crossings and energy just above the ESQPT critical energy and a larger one. In left column the squared energy components $\left|c_{k}\right|^{2}$ of the initial states $\left(\left|\alpha_{o}\right\rangle=\sum_{k} c_{k}\left|E_{k}\right\rangle\right)$ are shown

In all cases, quantum and classical results for the $S P$ (central column) and $j_{z}$ (right column) coincide until the Ehrenfest time, after this time the quantum results show larger oscillations than the classical approximations. However, a fairer comparison is obtained by considering rolling temporal averages, both, for the $S P$ and $j_{z}$. We observe that except in the first row, we obtain a remarkable classical-quantum correspondence, which indicates that in all these cases the dynamical tunneling is marginal and the quantum temporal trend is very well described classically. On the contrary, for the first row the classical-quantum correspondence is broken and the classical survival probability and $j_{z}$ are larger than the quantum ones. These are additional indications that the avoided crossings favor the dynamical tunneling if the initial coherent state is chosen on a classical trajectory with energy slightly above the critical energy of the ESQPT.

Note that in the case of the coherent state of the first row, all the energy components in the relevant energy interval participate in the initial state, whereas in the other cases we obtain components very close to zero that alternate (for a given parity) with components different to zero. This is understandable because in the case of the state of the first row, the energy eigenstates contributing the most to the initial state are located in a energy region just above the ESQPT energy where a superposition of the two classical trajectories is obtained. On the other hand, for the other cases, the Husimi function of the eigenstates are located either on one trajectory or other in an alternating way.

In all cases shown in Fig. 8, the initial coherent state is located on the outer trajectory of the pair of degenerate classical trajectories, and in the case of the state of the first row the tunneling occurs into the inner trajectory of the degenerate pair. In Appendix E, we confirm that similar results to those in Fig. 8 are obtained for initial coherent states located in the inner trajectory of the degenerate pair. Likewise, in Appendix E we show that for initial states with energy below the ESQPT and energies above $E / J=-1$, a classical-quantum correspondence is obtained even if the couplings fulfill the condition for avoided crossings.

\section{CONCLUSIONS}

For a particular region of the parameter space in the Lipkin-Meshkov-Glick model $\left(\gamma_{x}<-1\right.$ and $\left.\gamma_{y}<-1\right)$ there exists an intermediate energy region delimited by a logarithmic divergence and a discontinuity in the energy density of states, with the former defining a so-called excited-state quantum phase transition (ESQPT). At this intermediate energy region, real and avoided crossings occur for coupling parameters fulfilling a condition that was derived semi-classically. The real and avoided crossings were linked to the existence of pairs of different classical trajectories for the same energy that are not connected by the discrete parity symmetry of the model. Furthermore, the ESQPT was linked to the presence of a pair of hyperbolic fixed points in the phase space of the classical model.

We studied the avoided crossings appearing at this intermediate region through the Wehrl entropy, which is a measure of the phase space volume occupied by the quantum states. We found, as a function of the coupling parameters, a sudden increase and interchange of the Wehrl entropy for eigenstates participating in avoided crossings and having energy close to the critical ESQPT energy. For avoided crossings far enough of the ESQPT energy, the sudden increase of the Wehrl entropy disappears and only a simple interchange is observed. It was shown that the spike-like behaviour of the Wehrl entropy for avoided crossing close to the ESQPT comes from a superposition of the degenerate classical trajectories in the Husimi (phase-space) representation of the eigenstates involved in the avoided crossings; this superposition is absent in higher energy avoided crossings. The superposition implies a sudden augmentation of the phase space occupied by the states, and therefore an increase in the Wehrl entropy.

The singular behavior of the Wehrl entropy in avoided crossings for eigenstates close to the ESQPT energy enhance the dynamical tunneling between classically disconnected phase-space regions for non-stationary states having large components of these Hamiltonian eigenstates. It was also shown the way as this dynamical tunneling associated to avoided crossings induces a breaking of the quantum-classical correspondence in the temporal evolution of observables. This quantum-classical breaking occurs in the model, exclusively for states possessing large components of eigensates participating in the avoided crossings close to the ESQPT critical energy. For other non-stationary states the correspondence is kept.

The mechanism of quantum tunneling and classicalquantum breaking identified here for the LipkinMeshkoc-Glick model, could be also present in other quantum systems. Not only for one-degree-of-freedom models with pairs of hyperbolic fixed points, but also in models with more degrees of freedom where hyperbolic fixed points appears when the integrability of the models is broken and they move toward a chaotic regime [37]. The results presented here could also be relevant in the study of dynamical phase transitions [38], where avoided crossings and closeness to hyperbolic fixed points could influence the dynamics of non-stationary states in a similar way as we identified here for coherent states in the Lipkin-Meshkov-Glick model. 


\section{ACKNOWLEDGMENTS}

SL-H acknowledges useful discussions with M. Bastarrachea, J. Hirsch, S. Pilatowsky, L. Santos and D. Villaseñor with whom several techniques used in the paper were developed. DJN acknowledges financial support from PRODEP project number 42027 UV-CA-320 (Mexico), and SL-H from the Mexican CONACyT project CB2015-01/255702.

\section{Appendix A: Numerical methods}

We wrote specialized codes in fortran90 to calculate the spectrum of the Hamiltonian (1) and the Husimi representations. We performed the numerical diagonalization of the Hamiltonian (1) by using the subroutine dsyev of the Lapack package [39]. Once we had the eigenvalues and eigenstates we proceeded to calculate the Husimi representation (12) on a grid of canonical variables $Q$ and $P$. With the eigenvalues and eigenstates of the Hamiltonian, we also computed the evolution of the initial coherent state (14), the survival probability (16) and $\langle J\rangle(t)$ (17). For the entropy (13) we used Monte Carlo sampling of the Husimi representation (12).

For the line integral (15) along the classical trajectory, we divided the trajectory in small pieces, we evaluated (14) in each segment and finally we added the contributions from all circular segment i.e. numerical integration in polar coordinates. In order to calculate the classical survival probability and $j_{z}(t)$, we solved numerically the equations of motion corresponding to the Hamiltonian formalism in canonical variables $Q-P$ by using the software Mathematica.

\section{Appendix B: Energy density of states}

A classical approximation to the energy density of states (EDoS) can be obtained from the lowest order term of the Gutzwiller trace formula

$$
\rho_{s c}(E)=\frac{J}{2 \pi} \int d z d \phi \delta(H(z, \phi)-E),
$$

where $H(z, \phi)$ is given in Eq. 8. To evaluate the previous integral, we use the properties of the Dirac delta to obtain

$$
\begin{aligned}
\rho_{s c}(E)= & \frac{1}{2 \pi} \int_{\phi \in \boldsymbol{\Phi}_{\epsilon}} \frac{d \phi}{\sqrt{1-A(\phi)(2 \epsilon-A(\phi))}} \times \\
& \int_{-1}^{1} d z\left[\delta\left(z-z_{+}\right)+\delta\left(z-z_{-}\right)\right],
\end{aligned}
$$

where

$$
A(\phi)=\gamma_{x} \cos ^{2} \phi+\gamma_{y} \sin ^{2} \phi
$$

$$
z_{ \pm}(\phi, E)=\frac{1 \pm \sqrt{1-A(\phi)[2 \epsilon-A(\phi)]}}{A(\phi)}
$$

and $\boldsymbol{\Phi}_{\epsilon}$ is the set of values of $\phi$ for which there exist at least a solution of equation $H(z, \phi) / J=\epsilon$ for variable $z \in[-1,1]$, with $\epsilon=E / J$. In Fig. 9, we show $H(z, \phi) / J$ as a function of $z$ for different angles $\phi$ and for the same four set of couplings as in Fig. 1. The plots allow not only to visualize the range of allowed energies for given couplings, but also identify the different kind of trajectories that may appear in the different energy intervals. These latter are indicated by the same color code used in Fig. 1. Observe that the number of intersections of an horizontal line (constant $\epsilon=E / J$ ) with the quadratic curves $H(z, \phi) / J$ is equal to the second integral in Eq. (B2): for the blue region in II, blue and orange regions in III, and blue and green regions in IV, the number of intersection is two, while in the rest of regions this number is one. Equivalent expression for the EDoS were obtained in [22] by analyzing the zeros of the eigenstates Husimi functions.

\section{Appendix C: Condition for real and avoided crossings}

For sector III in parameter space and intermediate energy, there exist pairs of degenerate classical trajectories which are not connected by the parity symmetry of the model. One can obtain the set of energy levels associated to these two disconnected sets of classical orbits by considering the Einstein-Birllouin-Keller(EBK) quantization rule

$$
\frac{J}{2 \pi} \int_{-\pi}^{\pi} \tilde{z}_{ \pm}\left(\phi, E_{n_{ \pm}}\right) d \phi=\left(n_{ \pm}+\frac{1}{2}\right)
$$

where $n_{ \pm}$is an integer and $\tilde{z}_{ \pm}(\phi, E)$ are the two different classical trajectories for the same energy, $E$, expressed in terms of the canonical variables defined in Eq. (7). These trajectories are obtained from the quantum Hamiltonian (4) by mapping the pseudospin operators to classical vectors $\hat{J}_{i} \rightarrow j_{i}$ and expressing $z$ as a function of $\phi$ and $\hat{H} \rightarrow E$,

$$
\tilde{z}_{ \pm}(\phi, E)=\frac{1}{\tilde{A}(\phi)} \pm \frac{\sqrt{1-\tilde{A}(\phi)[2 \epsilon-\tilde{A}(\phi)]}}{\tilde{A}(\phi)}
$$

with

$$
\tilde{A}(\phi)=\frac{2 J}{2 J-1}\left(\gamma_{x} \cos ^{2} \phi+\gamma_{y} \sin ^{2} \phi\right)=\frac{2 J}{2 J-1} A(\phi)
$$

and $\epsilon=E / J$. Notice that $\tilde{z}$ and $\tilde{A}$ are very similar to the functions defined in Eqs. (B3) and (B4).

If two energy levels coincide $E_{n_{+}}=E_{n_{-}}$, by summing 
I

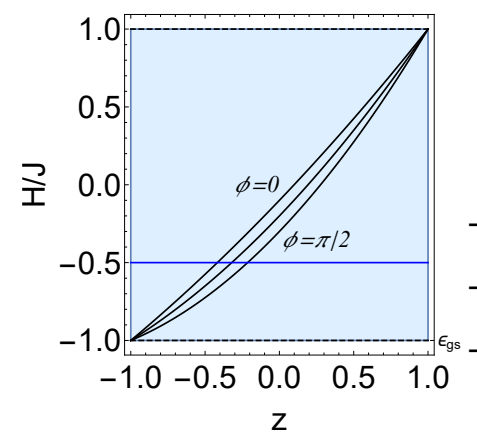

II

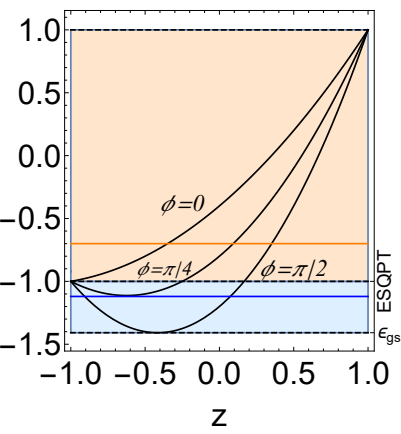

III

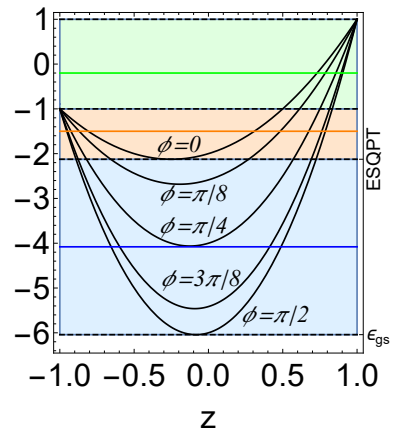

IV

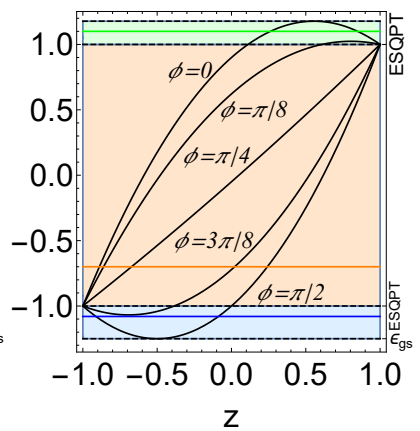

Figure 9: Solid black lines depict the classical energy $H(z, \phi) / J$ plotted as a function of $z$ for representative values of $\phi \in[0, \pi / 2]$. The value $\phi$ used in each line is indicated, but each line is actually associated to four different angles $( \pm \phi, \pi \pm \phi)$. The same sets of couplings $\left(\gamma_{x}, \gamma_{y}\right)$ as in Fig.1 were used, which are representative of the sectors I-IV in parameter space. Horizontal solid lines are representative energies of the different regimes, classified according to the

kind of trajectories $z(E, \phi)$ that can be obtained by considering the intersection of the horizontal lines with the quadratic black lines. We use the same colour code as in Fig.1 for indicating the different energy regions. Dashed horizontal lines indicate benchmarking energies, ground-state energies, ESQPT energies and $E / J=-1$ in the case of panel III.

equations $(\mathrm{C} 1)$ for both trajectories $\tilde{z}_{ \pm}$, we obtain

$$
\frac{J}{\pi} \int_{-\pi}^{\pi} \frac{d \phi}{\tilde{A}(\phi)}=n_{+}+n_{-}+1
$$

which after performing the integral leads to

$$
\frac{2 J-1}{\sqrt{\gamma_{x} \gamma_{y}}}=n_{+}+n_{-}+1 .
$$

This expression can be written as

$$
\gamma_{x} \gamma_{y}=\left(\frac{2 J-1}{p}\right)^{2},
$$

where $p=n_{+}+n_{-}+1>0$ is a positive integer number. Since the intermediate regime with pairs of disconnected trajectories for the same energy appear only in the region $\left|\gamma_{x}\right| \geq 1$ and $\left|\gamma_{y}\right| \geq 1$, the integer $p$ must be less or equal than $2 J-1$. Then the condition for having crossing of EBK levels can be written as

$$
\gamma_{x} \gamma_{y}=\left(\frac{2 J-1}{2 J-N}\right)^{2}
$$

with $N$ an integer satisfying $0<N<2 J$. We have verified that for $N$ odd crossings between states of different parity appear, whereas for $N$ even the crossing of the EBK energy levels become avoided crossings between states of same parity.

\section{Appendix D: Classical approximation to SP and $j_{z}$}

\section{Survival probability}

Following Ref.[36], we express the survival probability of an initial Bloch coherent state $S P(t)=\left|\left\langle\alpha_{o}|\hat{U}(t)| \alpha_{o}\right\rangle\right|^{2}$ in terms of the Wigner functions at times 0 and $t$,

$$
S P(t)=\frac{2 \pi}{J} \int d \mathbf{u} w_{\alpha_{o}}(\mathbf{u}, 0) w_{\alpha_{o}}(\mathbf{u}, t),
$$

where $\mathbf{u}=(Q, P)$ is a point in the phase space. The Wigner distribution for a Bloch coherent state is in turn given in terms of Legendre polynomials [40]

$$
\begin{aligned}
& w_{\alpha_{o}}(\theta, \phi)= \\
& \frac{(2 J) !}{4 \pi} \sum_{k=0}^{2 J} \sqrt{\frac{2 k+1}{(2 j-k) !(2 j+k+1) !}} P_{k}(\cos \Theta),
\end{aligned}
$$

where $\Theta$ is the angle between $\{\theta, \phi\}$ and $\left\{\theta_{0}, \phi_{0}\right\}$ i.e.

$$
\Theta=\cos \theta \cos \theta_{0}+\sin \theta \sin \theta_{0} \cos \left(\phi-\phi_{0}\right),
$$

with $\theta$ and $\phi$ angular spherical coordinates on the Bloch sphere. For large $J$, the Wigner distribution (D2) is well approximated by a normal distribution

$$
w_{\alpha_{0}}(\theta, \phi) \approx \frac{J}{\pi} e^{-J \Theta^{2}} .
$$

The Wigner function of the evolved state is obtained by using the truncated Wigner approximation (TWA), which consists of assuming that the evolution of the 
(e)

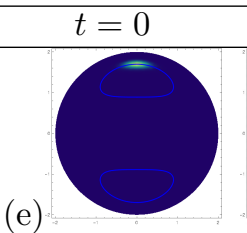

(f)

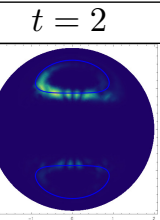

$\longrightarrow$
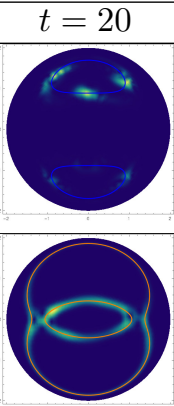
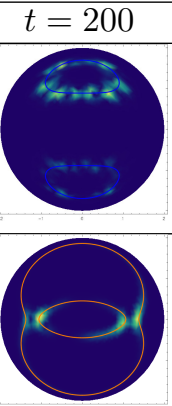

(g)

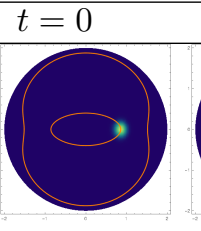

$\infty$

(

(h)

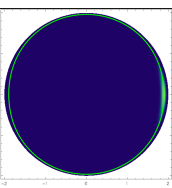

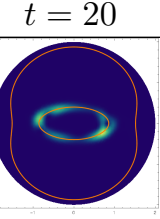

$t=50$

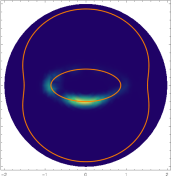

$\square$

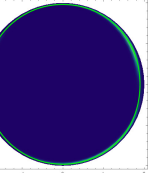

Figure 10: Density plots of the evolution of the Husimi functions $\mathcal{Q}_{\alpha_{0}}(\alpha, t)$ at selected times $t$ for four different initial Bloch coherent states. Solid orange line are classical trajectory at the same energy of the respective initial state $\left\langle\alpha_{o}|\hat{H}| \alpha_{o}\right\rangle=H$. In all panels the coupling parameters are chosen at the avoided crossing condition $\gamma_{x}^{A C}=-4.10331$ (with $\gamma_{y}^{A C}=3 \gamma_{x}^{A C}$ and $J=100$ ). In panel (e) the initial state has an energy well below the ESQPT. In (f) the initial state has an energy just above the ESQPT and is located in the inner trajectory of the degenerate pair. In $(\mathrm{g})$ the initial state has an energy in the intermediate regime but far enough from the ESQPT and is also located in the inner trajectory of the degenerate pair. In (h) the initial state has an energy in the high energy regime well above $E / J=-1$.
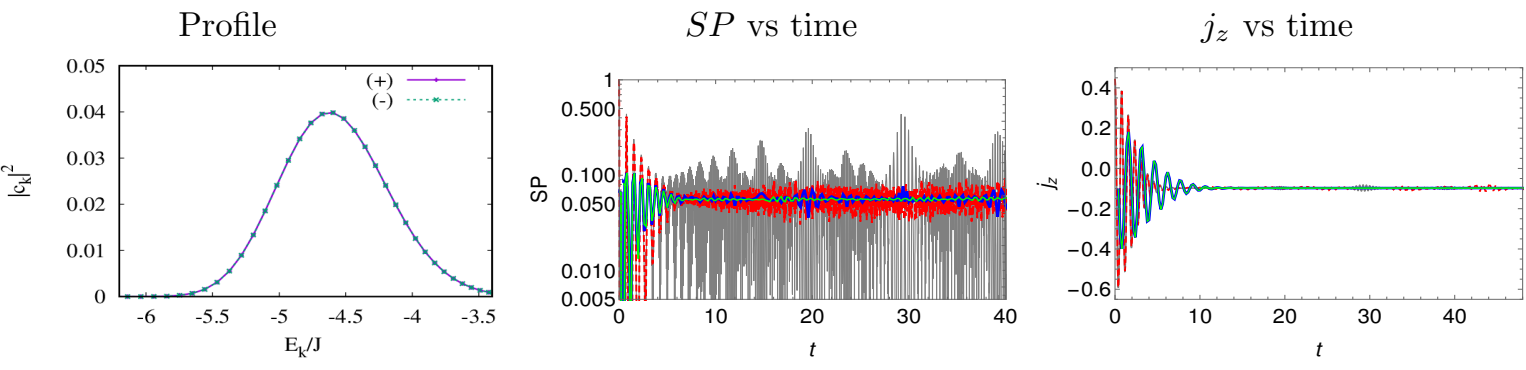

(e)

(f)
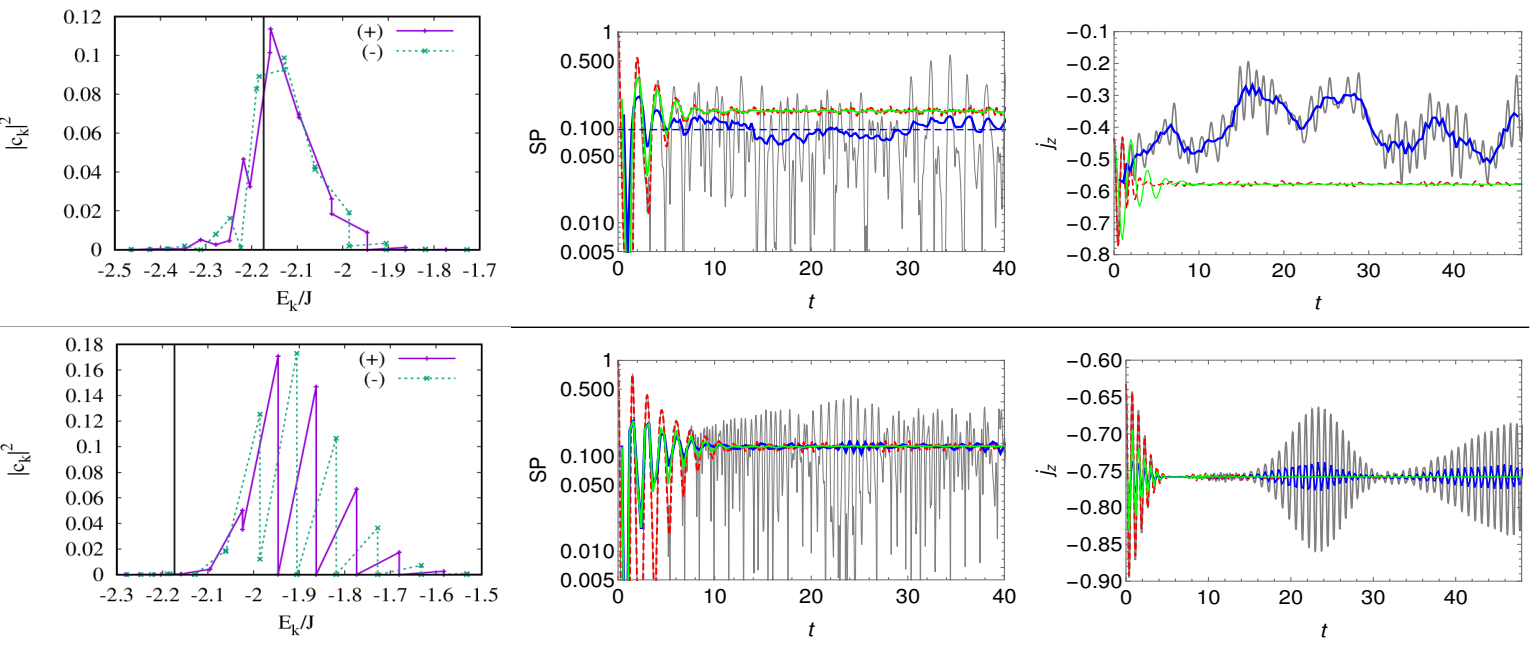

(g)
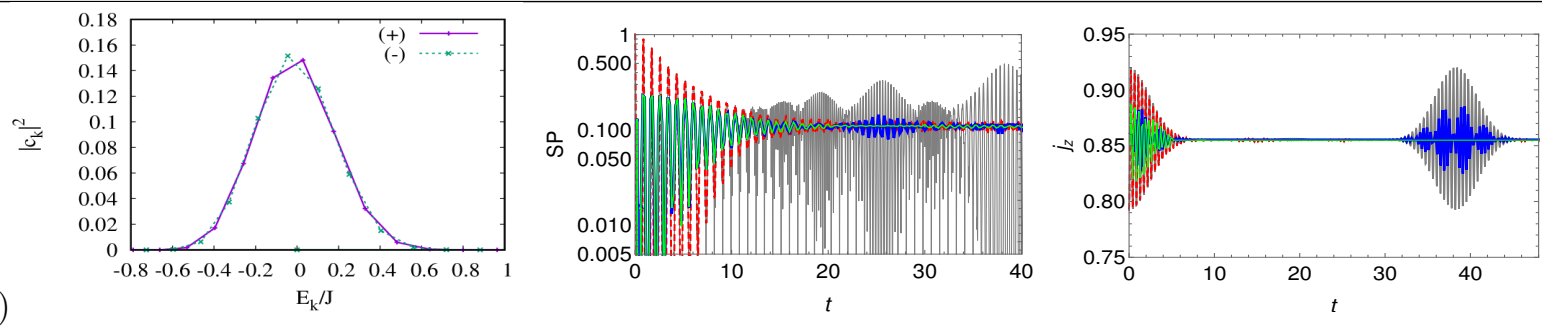

Figure 11: Same as Fig. 8, but for the four initial states of Fig.10. Only in state (f) a breaking of the classical-quantum correspondence is observed in the rolling averages of observables $S P$ and $j_{z}$. 
Wigner distribution is governed by the classical equations of motion $\frac{\partial w}{\partial t}=\{w, H\}$. From this we obtain $w(\mathbf{u}, t) \approx w\left(\varphi^{-t}(\mathbf{u}), 0\right)=w_{\alpha_{o}}\left(\varphi^{-t}(\mathbf{u})\right)$, where $\varphi$ is the function which describes the classical time evolution $\mathbf{u}(t)=\varphi^{t}\left(\mathbf{u}_{0}\right)$ of an initial condition $\mathbf{u}_{0}$. By using this result in the survival probability (D1) one gets the classical approximation,

$$
\begin{aligned}
S P(t) & \approx \frac{2 \pi}{J} \int d \mathbf{u} w(\mathbf{u}, 0) w\left(\varphi^{-t}(\mathbf{u}), 0\right) \\
& =\frac{2 \pi}{J} \int d \mathbf{u} w_{\alpha_{o}}(\mathbf{u}) w_{\alpha_{o}}\left(\varphi^{-t}(\mathbf{u})\right) .
\end{aligned}
$$

The previous integral can be viewed as the average of the Wigner function at time $t, w_{\alpha_{o}}^{(t)}$, weighted by the initial Wigner function $w_{\alpha_{o}}$, or, equivalently, as the average of the initial Wigner function weighted by the Wigner function at time $t$ :

$$
\left\langle w_{\alpha_{o}}\left(\varphi^{-t}(\mathbf{u})\right)\right\rangle_{w_{\alpha_{o}}}=\left\langle w_{\alpha_{o}}(\mathbf{u})\right\rangle_{w_{\alpha_{o}}^{(t)}} .
$$

From this, the integral (D4) can be estimated by means of Monte Carlo integration [36]

$$
S P(t) \approx \frac{1}{M} \sum_{i=1}^{M} w_{\alpha_{o}}\left(\varphi^{ \pm t}\left(\mathbf{u}_{i}\right)\right)
$$

where $\mathbf{u}_{i}$ are points randomly chosen following the initial distribution $w_{\alpha_{o}}$ and $M$ is the total number of Monte Carlo iterations.

\section{Temporal evolution of $\left\langle\hat{J}_{z}\right\rangle$}

The temporal evolution of the expectation value of the population operator can be calculated from the Wigner distribution

$$
j_{z}(t) \equiv \frac{1}{J}\left\langle\alpha_{0}\left|\hat{U}^{\dagger}(t) \hat{J}_{z} \hat{U}(t)\right| \alpha_{0}\right\rangle=\int d \mathbf{u} z(\mathbf{u}) w(u, t),
$$

where $z(\mathbf{u})=-\cos \theta=\left(\frac{1}{2}\left(Q^{2}+P^{2}\right)-1\right)$. Similar to the $S P$, we approximate classically this expression by using the TWA and the Gaussian distribution for the Wigner function of a coherent state (D3),

$$
j_{z}(t) \approx \int d \mathbf{u} w_{\alpha_{o}}\left(\varphi^{-t}(\mathbf{u})\right) z(\mathbf{u})
$$

The previous integral can be interpreted as the average of $z(\mathbf{u})$ weighted by the Wigner function at time $t\left(w_{\alpha_{o}}^{(t)}\right)$,

$$
j_{z}(t) \approx\langle z(\mathbf{u})\rangle_{w_{\alpha_{o}}^{(t)}} .
$$

To evaluate the integral, we also perform a Monte Carlo integration to obtain

$$
j_{z}(t) \approx \frac{1}{M} \sum_{i=1}^{M} z\left(\varphi^{t}\left(\mathbf{u}_{i}\right)\right)
$$

where $\mathbf{u}_{i}$ is a set of $M$ random points generated from the initial Wigner distribution (D3) and which evolve according to the classical Hamiltonian (6).

\section{Appendix E: Additional cases}

In this appendix we show the evolution of four different initial Bloch coherent states, for couplings satisfying the same condition of avoided crossing as in the vertical solid line in Fig.2(b). In Fig. 10 the evolution of the Husimi function $\mathcal{Q}_{\alpha_{0}}(\alpha, t)$ is presented. States (e) and (h) are chosen with energies outside the intermediate energy regime where avoided crossings occur. State (e) has an energy well below the ESQPT, whereas state (h) has an energy well above $E / J=-1$. Initial state (f) has an energy just above the ESQPT but, differently to state (a) in Fig. 7, it is initially located on the inner trajectory and tunneling takes place into the outer trajectory. The initial state (g) has an energy in the intermediate regime but far enough from the ESQPT, its Husimi function is located on the inner trajectory and no tunneling is observed in its unitary evolution. The squared energy components of the initial states, their survival probability and the evolution of the expectation value $\left\langle\hat{J}_{z}\right\rangle$ are presented in Fig. 11. It is confirmed that only in the case of state (f) the classical-quantum correspondence is broken for the rolling averages of the considered observables.
[1] Christopher Jarzynski, H. T. Quan, and Saar Rahav, "Quantum-classical correspondence principle for work distributions," Phys. Rev. X 5, 031038 (2015).

[2] Giuliano Benenti and Giulio Casati, "Quantum-classical correspondence in perturbed chaotic systems," Phys. Rev. E 65, 066205 (2002).

[3] Meenu Kumari and Shohini Ghose, "Quantum-classical correspondence in the vicinity of periodic orbits," Phys. Rev. E 97, 052209 (2018).

[4] Pavel Cejnar, Pavel Stránský, Michal Macek, and Michal Kloc, "Excited-state quantum phase transitions," Jour- nal of Physics A: Mathematical and Theoretical 54, 133001 (2021).

[5] G. Engelhardt, V. M. Bastidas, W. Kopylov, and T. Brandes, "Excited-state quantum phase transitions and periodic dynamics," Phys. Rev. A 91, 013631 (2015).

[6] Francisco Pérez-Bernal Qian Wang, "Signatures of excited state quantum phase transitions in quantum many body systems: Phase space analysis," (2020), arXiv:2011.11932 [quant-ph].

[7] Saúl Pilatowsky-Cameo, Jorge Chávez-Carlos, Miguel A. Bastarrachea-Magnani, Pavel Stránský, Sergio Lerma- 
Hernández, Lea F. Santos, and Jorge G. Hirsch, "Positive quantum lyapunov exponents in experimental systems with a regular classical limit," Phys. Rev. E 101, 010202 (2020).

[8] Efim B. Rozenbaum, Sriram Ganeshan, and Victor Galitski, "Lyapunov exponent and out-of-time-ordered correlator's growth rate in a chaotic system," Phys. Rev. Lett. 118, 086801 (2017).

[9] J. von Neumann and E. P. Wigner, "über merkwürdige diskrete eigenwerte," Z. Phys. 30 30, 465 (1929).

[10] Clarence Zener and Ralph Howard Fowler, "Nonadiabatic crossing of energy levels," Proceedings of the Royal Society of London. Series A 137, 696-702 (1932).

[11] Landau L.D., "9 - a theory of energy transfer. ii," in Collected Papers of L.D. Landau, edited by D. Ter Haar (Pergamon, 1965) pp. 63-66.

[12] M Wilkinson, "Narrowly avoided crossings," Journal of Physics A: Mathematical and General 20, 635-645 (1987).

[13] Kyu-Won Park, Songky Moon, Younghoon Shin, Jinuk Kim, Kabgyun Jeong, and Kyungwon An, "Shannon entropy and avoided crossings in closed and open quantum billiards," Phys. Rev. E 97, 062205 (2018).

[14] F. J. Arranz, R. M. Benito, and F. Borondo, "Shannon entropy at avoided crossings in the quantum transition from order to chaos," Phys. Rev. E 99, 062209 (2019).

[15] H.J. Lipkin, N. Meshkov, and A.J. Glick, "Validity of many-body approximation methods for a solvable model: (i). exact solutions and perturbation theory," Nuclear Physics 62, 188-198 (1965).

[16] R. Botet, R. Jullien, and P. Pfeuty, "Size scaling for infinitely coordinated systems," Phys. Rev. Lett. 49, 478481 (1982).

[17] R. G. Unanyan and M. Fleischhauer, "Decoherence-free generation of many-particle entanglement by adiabatic ground-state transitions," Phys. Rev. Lett. 90, 133601 (2003).

[18] Jon Links, Huan-Qiang Zhou, Ross H McKenzie, and Mark D Gould, "Algebraic bethe ansatz method for the exact calculation of energy spectra and form factors: applications to models of bose einstein condensates and metallic nanograins," Journal of Physics A: Mathematical and General 36, R63-R104 (2003).

[19] Gang Chen, J. Q. Liang, and Suotang Jia, "Interactioninduced lipkin-meshkov-glick model in a bose-einstein condensate inside an optical cavity," Opt. Express 17, 19682-19690 (2009).

[20] Octavio Castaños, Ramón López-Peña, Jorge G. Hirsch, and Enrique López-Moreno, "Classical and quantum phase transitions in the lipkin-meshkov-glick model," Phys. Rev. B 74, 104118 (2006).

[21] Julien Vidal, José M. Arias, Jorge Dukelsky, and José Enrique García-Ramos, "Scalar two-level boson model to study the interacting boson model phase diagram in the casten triangle," Phys. Rev. C 73, 054305 (2006).

[22] Pedro Ribeiro, Julien Vidal, and Rémy Mosseri, "Exact spectrum of the lipkin-meshkov-glick model in the thermodynamic limit and finite-size corrections," Phys. Rev. E 78, 021106 (2008).

[23] A. Relaño, J. M. Arias, J. Dukelsky, J. E. García-Ramos, and P. Pérez-Fernández, "Decoherence as a signature of an excited-state quantum phase transition," Phys. Rev. A 78, 060102 (2008).
[24] Ricardo Puebla, Armando Relaño, and Joaquín Retamosa, "Excited-state phase transition leading to symmetry-breaking steady states in the dicke model," Phys. Rev. A 87, 023819 (2013).

[25] R. González-Férez and J. S. Dehesa, "Shannon entropy as an indicator of atomic avoided crossings in strong parallel magnetic and electric fields," Phys. Rev. Lett. 91, 113001 (2003).

[26] He Y. L., Chen Y., Han J. N., Zhu Z. B., Xiang G. X., Liu H. D., Ma B. H., and He D. C., "Shannon entropy as an indicator of atomic avoided crossings for rydberg potassium atoms interacting with a static electric field," Eur. Phys. J. D 69, 283 (2015).

[27] Octavio Castaños, Manuel Calixto, Francisco PérezBernal, and Elvira Romera, "Identifying the order of a quantum phase transition by means of wehrl entropy in phase space," Phys. Rev. E 92, 052106 (2015).

[28] Elvira Romera, Octavio Castaños, Manuel Calixto, and Francisco Pérez-Bernal, "Delocalization properties at isolated avoided crossings in lipkin-meshkov-glick type hamiltonian models," Journal of Statistical Mechanics: Theory and Experiment 2017, 013101 (2017).

[29] Sven Gnutzmann and Karol Zyczkowski, "Rényi-wehrl entropies as measures of localization in phase space," Journal of Physics A: Mathematical and General 34, 10123-10139 (2001).

[30] A D Ribeiro, M A M de Aguiar, and A F R de Toledo Piza, "The semiclassical coherent state propagator for systems with spin," Journal of Physics A: Mathematical and General 39, 3085-3097 (2006).

[31] Pavel Stránský, Michal Macek, and Pavel Cejnar, "Excited-state quantum phase transitions in systems with two degrees of freedom: Level density, level dynamics, thermal properties," Annals of Physics 345, 73-97 (2014).

[32] Octavio Castaños, Ramón López-Peña, Jorge G. Hirsch, and Enrique López-Moreno, "Phase transitions and accidental degeneracy in nonlinear spin systems," Phys. Rev. B 72, 012406 (2005).

[33] S. Lerma H. and J. Dukelsky, "The lipkin-meshkov-glick model as a particular limit of the su(1,1) richardson-gaudin integrable models," Nuclear Physics B 870, 421-443 (2013).

[34] Alfred Wehrl, "General properties of entropy," Rev. Mod. Phys. 50, 221-260 (1978).

[35] D. Villaseñor, S. Pilatowsky-Cameo, M. A. BastarracheaMagnani, S. Lerma-Hernández, and J. G. Hirsch, "Quantum localization measures in phase space," Phys. Rev. E 103, 052214 (2021).

[36] D Villaseñor, S Pilatowsky-Cameo, M A BastarracheaMagnani, S Lerma-Hernández, L F Santos, and J G Hirsch, "Quantum vs classical dynamics in a spin-boson system: manifestations of spectral correlations and scarring," New Journal of Physics 22, 063036 (2020).

[37] F. J. Arranz, F. Borondo, and R. M. Benito, "Scar formation at the edge of the chaotic region," Phys. Rev. Lett. 80, 944-947 (1998).

[38] R. J. Lewis-Swan, S. R. Muleady, D. Barberena, J. J. Bollinger, and A. M. Rey, "Characterizing the dynamical phase diagram of the dicke model via classical and quantum probes," Phys. Rev. Research 3, L022020 (2021).

[39] E. Anderson, Z. Bai, C. Bischof, S. Blackford, J. Demmel J. Dongarra, J. Du Croz, A. Greenbaum, S. Hammarling, A. McKenney, and D. Sorensen, LAPACK Users' 
Guide, 3rd ed. (SIAM, Philadelphia, Pennsylvania, USA, 1999).

[40] Popo Yang, Iván F Valtierra, Andrei B Klimov, ShinTza Wu, Ray-Kuang Lee, Luis L Sánchez-Soto, and Gerd
Leuchs, "The wigner flow on the sphere," Physica Scripta 94, 044001 (2019) 